Nanolaminate Deformable Mirrors

A. P. Papavasiliou, S. S. Olivier, T. W. Barbee, R.

R. Miles, C. C. Walton, M. B. Cohn, K. Chang

June 2, 2006

Optical MEMS 2006

Big Sky, MT, United States

August 21, 2006 through August 24, 2006 
This document was prepared as an account of work sponsored by an agency of the United States Government. Neither the United States Government nor the University of California nor any of their employees, makes any warranty, express or implied, or assumes any legal liability or responsibility for the accuracy, completeness, or usefulness of any information, apparatus, product, or process disclosed, or represents that its use would not infringe privately owned rights. Reference herein to any specific commercial product, process, or service by trade name, trademark, manufacturer, or otherwise, does not necessarily constitute or imply its endorsement, recommendation, or favoring by the United States Government or the University of California. The views and opinions of authors expressed herein do not necessarily state or reflect those of the United States Government or the University of California, and shall not be used for advertising or product endorsement purposes. 


\title{
Nanolaminate Deformable Mirrors
}

\author{
Alexandros P. Papavasiliou ${ }^{\mathrm{a}}$, Scot S. Olivier ${ }^{\mathrm{a}}$, Troy W. Barbee ${ }^{\mathrm{a}}$, Robin R. Miles ${ }^{\mathrm{a}}$, Chris C. \\ Walton ${ }^{\mathrm{a}}$, Michael B. Cohn ${ }^{\mathrm{b}}$, and Kevin Chang ${ }^{\mathrm{b}}$ \\ ${ }^{a}$ Lawrence Livermore National Laboratory, 7000 East Ave., Livermore, CA 94550 \\ ${ }^{b}$ MicroAssembly Technologies, 3065 Richmond Parkway, Suite 109, Richmond, CA 94806
}

\section{INTRODUCTION}

LLNL is developing nanolaminate-based deformable mirrors for terrestrial and spacebased optical systems. We are combining two complementary technologies: high-spatialdensity electrostatic actuators and thin, flexible, lightweight nanolaminate foils. Electrostatic actuation of MEMS-like structures provides densely-spaced, repeatable deflections on the order of $10 \mu \mathrm{m}$. Nanolaminate foils provide a mirror surface that is simultaneously flexible enough to deform under electrostatic forces and tough enough to survive handling and bonding.

We are working on two similar deformable mirrors that will demonstrate the feasibility of nanolaminate-based deformable mirrors over a wide range of size scales. The high-density device has a pitch of $1.255 \mathrm{~mm}$ between 1,024 square pixels. The large-scale device has a pitch of $9 \mathrm{~mm}$ between 76 hexagonal pixels [1], [2].

\section{Nanolaminate Foils}

Nanolaminate foils are a new class of materials under developed at LLNL [3]. They are produced by sputtering alternating thin layers of alternating materials. Each layer is on the order of a few nanometers thick, but thousands of layers are deposited to build up foils $1-100 \mu \mathrm{m}$ in thickness. When the foil is released from the parting-layer-coated mandrel, the foil duplicates the mandrel's high-quality surface figure. The fabrication process produces a nano-structured material with very small grains and a high dislocation density, which results in very high strength and toughness. This strength and toughness make it possible to fabricate foils that are simultaneously strong enough to withstand handling and bonding and thin and flexible enough to be deformed by electrostatic actuators.

\section{DeVice Structure}

Both devices use the same basic structure, modified to fit the size requirements. We create a MEMS-like structure with technologies that are scalable from the centimeter- to meter-diameter devices. The vertical vias that separate layers in a MEMS electrostatic actuator are replaced with electroplated metal ridges and posts. The horizontal silicon layers in a MEMS actuator are replaced with nanolaminate layers. The nanolaminate actuator layer is attached to metal ridges that suspend it above electrodes on the surface. A set of metal posts is electroplated on the back side of the mirror layer and is used to attach the mirror to the actuator layer.

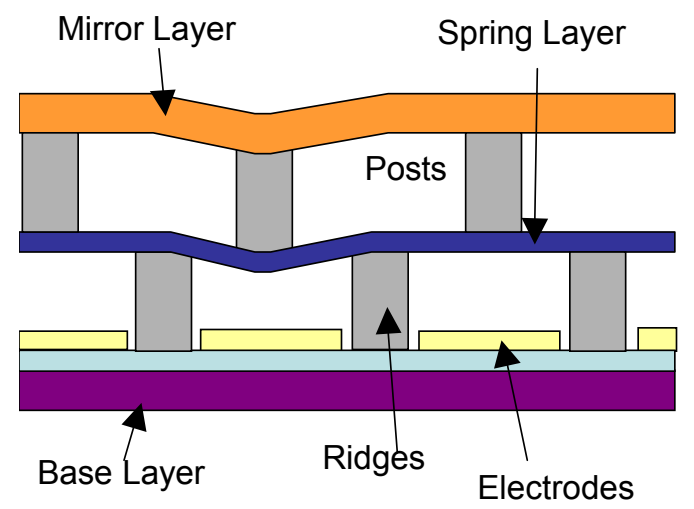

Figure 1. Cross-sectional view of the deformable mirrors. The grounded spring layer is deformed by electrostatic attraction to electrodes on the base layer. The motion of the spring layer is translated to the mirror through a set of posts.

This design architecture is highly scalable for different pixel sizes. The accuracy of the deflection of the mirror is proportional to the vertical dimensions. The vertical dimensions are all controlled by thicknesses of deposited materials. Since it is possible to produce electroplated structures and nanolaminate foils with well-controlled thicknesses on the order of the desired deflections, it is possible to produce a mirror with well-controlled deflection.

The sensitivity of this type of structure to line-width errors is proportional to the pitch between pixels. The high-density devices are produced with conventional microlithography with line-width errors on the order of a micron. The large-scale device is tolerant of line-width errors on the order of $50 \mu \mathrm{m}$, allowing the parts to be defined by conventional printed-circuitboard lithography techniques that can be used on the meter scale. 


\section{FABRICATION AND TESTING}

The base layers for all the prototype deformable mirrors reported here are fabricated from oxidized silicon wafers. Gold electrodes are patterned on the surface and nickel ridges are electroplated. Nickel posts are electroplated on the bottom side of the nanolaminate mirror layers. The spring layer is bonded to the ridges on the base layer and the posts are bonded to the spring layer with epoxy [4].

Complete 76-pixel large-scale devices were assembled by MicroAssembly Technologies from base layers and nanolaminate foils fabricated at Lawrence Livermore National Laboratory. Preliminary testing has measured the deflection of both pixels in the middle of the array as well as pixels at the edge of the mirror, which are less constrained. The middle pixels have maximum deflection of $1.5 \mu \mathrm{m}$ and the edge pixels deflect over $4 \mu \mathrm{m}$.

Spring layers have been bonded to base layers for the $32 \times 32$ pixel high-density device and the deflection of the spring layer has been measured to be as high as $3.6 \mu \mathrm{m}$.

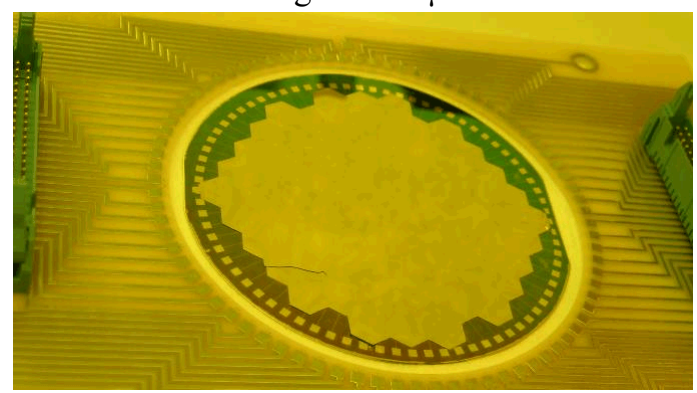

Figure 3. A 76-pixel large-scale nanolaminate deformable

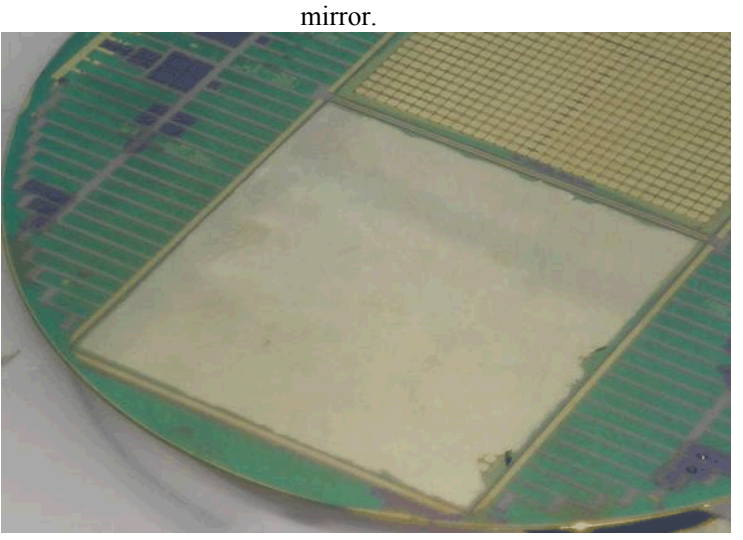

Figure 4. The spring layer of a $32 \times 32$-pixel high-density nanolaminate deformable mirror.

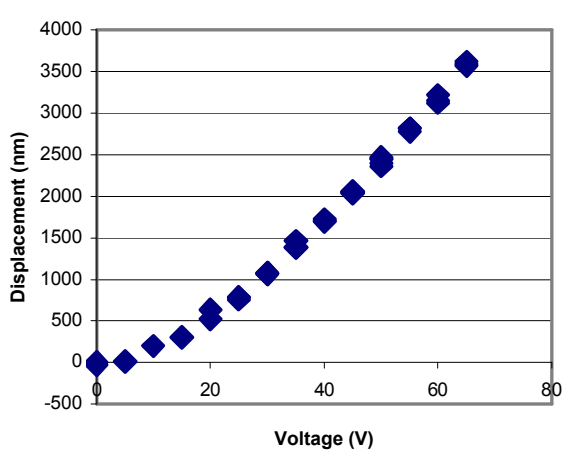

Figure 4. Deflection vs. voltage for the spring layer of a $32 \mathrm{x}$ 32 high-density mirror.

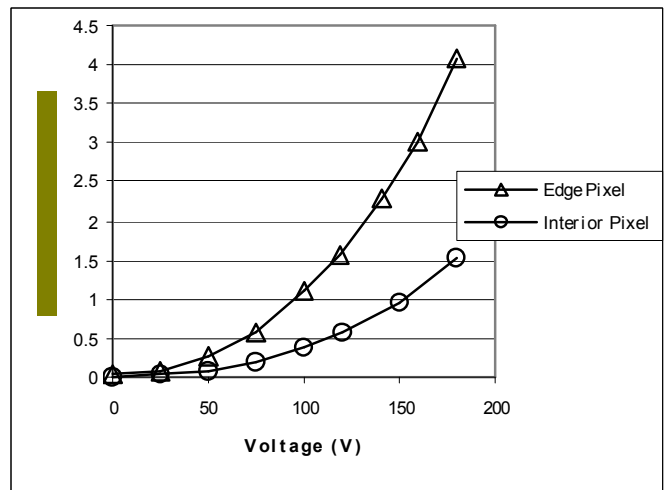

Figure 5. Deflection vs. voltage for the 76-pixel large-scale deformable mirror.

\section{ACKNOWLEDGMENT}

This work was performed under the auspices of the U.S. Department of Energy by University of California, Lawrence Livermore National Laboratory under Contract W-7405-Eng-48.

\section{REFERENCES}

[1] A.P. Papavasiliou S.S. Olivier, C.C. Walton, M.B. Cohn, "MEMS actuated deformable mirror," Proc. SPIE, vol. 6113, pp. 190-199, Jan. 2006.

[2] A.P. Papavasiliou S.S. Olivier, T.W. Barbee, R.R. Miles, K. Chang, "Large-scale nanolaminate deformable mirror," Proc. SPIE, vol. 6113, pp. 200-209, Jan. 2006.

[3] T. W. Barbee, Jr., "Nano-Laminates: A New Class of Materials for Aerospace Applications", 0-7803-7651$\mathrm{X} / 03 / \$ 17.00$ (C) 2003 IEEE

[4] M. Cohn, K. Chang, A. Papavasiliou, S. Olivier, G. Kim, G. Matus, Z. Xiao, S. Cheung, K. Saechao, and R. Roehnelt, "High performance adaptive optics using microscale assembly," Proc. SPIE, vol. 6113, pp. 162$170,2006$. 